\title{
On a nonlocal problem for fractional differential equations via resolvent operators
}

\author{
Lizhen Chen ${ }^{1}$, Zhenbin Fan ${ }^{2 *}$ and Gang Li ${ }^{3}$
}

${ }^{*}$ Correspondence:

jsmathfan@gmail.com

${ }^{2}$ Department of Mathematics, Changshu Institute of Technology,

Suzhou, Jiangsu 215500, P.R. China

Full list of author information is

available at the end of the article

\begin{abstract}
Using the techniques of approximate solutions, the analytic resolvent method, and the uniform continuity of the resolvent, we discuss the existence of mild solutions for nonlocal fractional differential equations governed by a linear closed operator which generates a resolvent. An example is also given to illustrate the application of our theory.
\end{abstract}

MSC: $34 \mathrm{~K} 37 ; 47 \mathrm{~A} 10$

Keywords: nonlocal conditions; analytic resolvent; Caputo fractional order derivative; mild solution

\section{Introduction}

In this paper, we are concerned with the existence of mild solutions for the following fractional differential equation:

$$
\begin{aligned}
& D^{\alpha} u(t)=A u(t)+J_{t}^{1-\alpha} f(t, u(t)), \quad t \in J=[0, b], \\
& u(0)=g(u),
\end{aligned}
$$

where $D^{\alpha}$ is the Caputo fractional derivative of order $\alpha$ with $0<\alpha<1, A: D(A) \subset X \rightarrow X$ is the infinitesimal generator of a resolvent $S_{\alpha}(t), t \geq 0, X$ is a real Banach space, $J_{t}^{1-\alpha} h$ denotes the $1-\alpha$ order fractional integral of $h \in L^{1}([0, b], X) . f$ and $g$ are appropriate continuous functions to be specified later.

Byszewski and Lakshmikantham [1] introduced nonlocal initial conditions into the initial-value problems and argued that the corresponding models more accurately describe the phenomena since more information was taken into account at the onset of the experiment, thereby reducing the ill effects incurred by a single initial measurement. Concerning the motivations, relevant developments, and current status of the theory we refer the reader to [2-4]. We remark that the main difficulty in dealing with the nonlocal problem is how to get the compactness of the solution operator at zero. Many methods and techniques have been developed to avoid this difficulty in nonlocal problems, we refer the reader to the papers [5-9] and the references therein.

On the other hand, the theory of fractional differential equations has received much attention over the past 20 years, since they are important in describing the natural models such as diffusion processes, stochastic processes, finance and hydrology. Concerning 
the literature of fractional equations we refer to [10-18]. However, few authors have considered the fractional nonlocal problem (1.1) governed by a linear closed operator which generates a resolvent. The main difficulty is the resolvent does not have the property of semigroups, even the continuity in the uniform operator topology. Fortunately, we have proven the continuity of the resolvent in the uniform operator topology and given the characterization of compactness for resolvents in the case of an analytic resolvent. For more details, we refer the reader to the recent paper [19] by Fan.

In this paper, we study the existence of the nonlocal fractional differential equation (1.1) governed by operator $A$ generating an analytic resolvent. A standard approach in deriving the mild solution of (1.1) is define the solution operator $Q$. Then conditions are given such that some fixed point theorems such as Schauder's and Browder's can be applied to get a fixed point for solution operator $Q$, which gives rise to a mild solution of (1.1). As, the compactness or Lipschitz condition is required in this standard method. In this paper, to get rid of these restrictive conditions, based on the works of Fan and Li [4] and Zhu and Li [8], we mainly apply the techniques of approximate solutions, the analytic resolvent method and the uniform continuity of the resolvent to get the mild solution of the nonlocal fractional differential equation (1.1) without the compactness or Lipschitz continuity assumption on the nonlocal item $g$. Therefore, our results essentially generalize and improve many previous ones in this field [20-23].

The outline of this paper is as follows. In Section 2, we recall some definitions on Caputo fractional derivatives, analytic resolvent and the mild solutions to (1.1). In Section 3, we establish the existence of mild solutions of (1.1) when the nonlocal item is only continuous. An example is also given to illustrate our abstract results in the last section.

\section{Preliminaries}

Let $(X,\|\cdot\|)$ be a real Banach space. We denote $C([0, b], X)$ the space of all $X$-valued continuous functions on $[0, b]$ with the norm $\|u\|=\sup \{\|u(t)\|, t \in[0, b]\}, L^{p}([0, b], X)$ the space of $X$-valued Bochner integrable functions on $[0, b]$ with the norm $\|f\|_{L^{p}}=$ $\left(\int_{0}^{b}\|f(t)\|^{p} \mathrm{~d} t\right)^{1 / p}$, where $1 \leq p<\infty$. Also, we denote by $\mathscr{L}(X)$ the space of bounded linear operators from $X$ into $X$ endowed with the norm of operators.

Now let us recall some basic definitions and results on fractional derivatives and resolvents.

Definition 2.1 ([24]) Suppose $0<\alpha<1$. The fractional order integral of the function $f \in$ $L^{1}([0, b], X)$ of order $\alpha$ is defined by

$$
J_{t}^{\alpha} f(t)=\frac{1}{\Gamma(\alpha)} \int_{0}^{t}(t-s)^{\alpha-1} f(s) \mathrm{d} s
$$

where $\Gamma$ is the Gamma function.

Definition 2.2 ([24]) Suppose $0<\alpha<1$. The Caputo fractional order derivative of order $\alpha$ of a function $f \in L^{1}([0, b], X)$ given on the interval $[0, b]$ is defined by

$$
D^{\alpha} f(t)=\frac{1}{\Gamma(1-\alpha)} \int_{0}^{t}(t-s)^{-\alpha} f^{(1)}(s) \mathrm{d} s
$$


In the remainder of this paper, we always suppose that $0<\alpha<1, g_{\alpha}(t)=t^{\alpha-1} / \Gamma(\alpha), t>0$, and $A$ is a closed and densely defined linear operator on $X$.

Definition 2.3 ([25]) A family $\left\{S_{\alpha}(t)\right\}_{t \geq 0} \subseteq \mathscr{L}(X)$ of bounded linear operators in $X$ is called a resolvent (or solution operator) generated by $A$ if the following conditions are satisfied.

(S1) $S_{\alpha}(t)$ is strong continuous on $\mathbb{R}_{+}$and $S_{\alpha}(0)=I$;

(S2) $S_{\alpha}(t) D(A) \subseteq D(A)$ and $A S_{\alpha}(t) x=S_{\alpha}(t) A x$ for all $x \in D(A)$ and $t \geq 0$;

(S3) the resolvent equation holds:

$$
S_{\alpha}(t) x=x+\int_{0}^{t} g_{\alpha}(t-s) A S_{\alpha}(s) x \mathrm{~d} s \quad \text { for all } x \in D(A), t \geq 0
$$

Since $A$ is a closed and densely defined operator on $X$, it is easy to show that the resolvent equation holds for all $x \in X$ (see [25]).

For $\omega, \theta \in \mathbb{R}$, let

$$
\sum(\omega, \theta):=\{\lambda \in \mathbb{C}:|\arg (\lambda-\omega)|<\theta\} .
$$

Definition 2.4 ([25]) A resolvent $S_{\alpha}(t)$ is called analytic, if the function $S_{\alpha}(\cdot): \mathbb{R}_{+} \rightarrow \mathscr{L}(X)$ admits analytic extension to a sector $\sum\left(0, \theta_{0}\right)$ for some $0<\theta_{0} \leq \pi / 2$. An analytic resolvent $S_{\alpha}(t)$ is said to be of analyticity type $\left(\omega_{0}, \theta_{0}\right)$ if for each $\theta<\theta_{0}$ and $\omega>\omega_{0}$ there is $M_{1}=$ $M_{1}(\omega, \theta)$ such that $\|S(z)\| \leq M_{1} e^{\omega \operatorname{Re} z}$ for $z \in \sum(0, \theta)$, where $\operatorname{Re} z$ denotes the real part of $z$.

Definition 2.5 A resolvent $S_{\alpha}(t)$ is called compact for $t>0$ if for every $t>0, S_{\alpha}(t)$ is a compact operator.

Lemma 2.6 ([19, Lemmas 3.4, 3.5, 3.8]) Suppose $S_{\alpha}(t)$ is a compact analytic resolvent of analyticity type $\left(\omega_{0}, \theta_{0}\right)$. Then

(i) $\lim _{h \rightarrow 0}\left\|S_{\alpha}(t+h)-S_{\alpha}(t)\right\|=0$ for $t>0$;

(ii) $\lim _{h \rightarrow 0^{+}}\left\|S_{\alpha}(t+h)-S_{\alpha}(h) S_{\alpha}(t)\right\|=0$ for $t>0$;

(iii) $\lim _{h \rightarrow 0^{+}}\left\|S_{\alpha}(t)-S_{\alpha}(h) S_{\alpha}(t-h)\right\|=0$ for $t>0$.

Now, we consider the following fractional differential equation:

$$
\left\{\begin{array}{l}
D^{\alpha} x(t)=A x(t)+J_{t}^{1-\alpha} f(t), \quad 0<t \leq b, \\
x(0)=x_{0} .
\end{array}\right.
$$

Suppose that $x_{0} \in X, f \in L^{1}([0, b], X)$, and $x$ is a solution of (2.1). Then we can give the following variation of the constant formula:

$$
x(t)=S_{\alpha}(t) x_{0}+\int_{0}^{t} S_{\alpha}(t-s) f(s) \mathrm{d} s, \quad 0 \leq t \leq b .
$$

In fact, if $x$ satisfies (2.1), then for $0 \leq t \leq b$,

$$
x(t)=x_{0}+A\left(g_{\alpha} * x\right)(t)+\int_{0}^{t} f(s) \mathrm{d} s
$$


Thus, it follows from the definition of resolvent that

$$
\begin{aligned}
1 * x & =\left(S_{\alpha}-A g_{\alpha} * S_{\alpha}\right) * x=S_{\alpha} * x-S_{\alpha} *\left(A g_{\alpha} * x\right) \\
& =S_{\alpha} *\left(x_{0}+1 * f\right)=S_{\alpha} * x_{0}+1 * S_{\alpha} * f,
\end{aligned}
$$

which implies that $x(t)=S_{\alpha}(t) x_{0}+\int_{0}^{t} S_{\alpha}(t-s) f(s) \mathrm{d} s, 0 \leq t \leq b$.

So, we can give the following definition of mild solutions for (1.1).

Definition 2.7 A function $u \in C([0, b], X)$ is called a mild solution of fractional differential equation (1.1) if it satisfies

$$
u(t)=S_{\alpha}(t) g(u)+\int_{0}^{t} S_{\alpha}(t-s) f(s, u(s)) \mathrm{d} s, \quad 0 \leq t \leq b .
$$

\section{Existence results}

In this section, by using the techniques of approximate solutions, analytic resolvent and fixed point, we prove an existence theorem for the nonlocal problem (1.1) when the nonlocal item $g$ is only continuous in $C([0, b], X)$.

Let $r$ be a fixed positive real number. We consider the sets $B_{r}=\{x \in X:\|x\| \leq r\}, W_{r}=$ $\left\{x \in C([0, b], X): x(t) \in B_{r}, \forall t \in[0, b]\right\}$.

We list the following hypotheses:

(HA) $S_{\alpha}(t)$ is a compact analytic resolvent of analyticity type $\left(\omega_{0}, \theta_{0}\right)$ and $M=\sup _{t \in[0, b]}\left\|S_{\alpha}(t)\right\|<+\infty$.

(Hf) $f:[0, b] \times X \rightarrow X$ is a Caratheodory function, i.e., for a.e. $t \in[0, b], f(t, \cdot): X \rightarrow X$ is continuous and for all $x \in X$, the function $f(\cdot, x):[0, b] \rightarrow X$ is measurable. Moreover, for any $l>0$, there exists a function $\rho_{l} \in L^{1}([0, b], \mathbb{R})$ such that $\|f(t, x)\| \leq \rho_{l}(t)$ for a.e. $t \in[0, b]$ and all $x \in B_{l}$.

(Hg) $g: C([0, b], X) \rightarrow X$ is a continuous mapping, which maps $B_{r}$ into a bounded set and there is a $\delta=\delta(r) \in(0, b)$ such that $g(u)=g(v)$ for any $u, v \in W_{r}$ with $u(s)=v(s), s \in[\delta, b]$.

Theorem 3.1 Assume that (HA), (Hf), and (Hg) are satisfied. Then the nonlocal problem (1.1) has at least one mild solution on $[0, b]$ provided that

$$
M^{2} \sup _{u \in W_{r}}\|g(u)\|+M\left\|\rho_{r}\right\|_{L^{1}} \leq r .
$$

In the proof of the above theorem, we will need the following auxiliary result. For fixed $n \geq 1$, we consider the following approximate problem:

$$
\begin{aligned}
& D^{\alpha} u(t)=A u(t)+J_{t}^{1-\alpha} f(t, u(t)), \quad t \in J=[0, b], \\
& u(0)=S_{\alpha}\left(\frac{1}{n}\right) g(u) .
\end{aligned}
$$

Lemma 3.2 Assume that all the conditions in Theorem 3.1 are satisfied. Then for any $n \geq 1$, the nonlocal problem (3.1) has at least one mild solution $u_{n} \in C([0, b], X)$. 
Proof For fixed $n \geq 1$, set $Q_{n}: C([0, b], X) \rightarrow C([0, b], X)$ defined by

$$
\left(Q_{n} u\right)(t)=S_{\alpha}(t) S_{\alpha}\left(\frac{1}{n}\right) g(u)+\int_{0}^{t} S_{\alpha}(t-s) f(s, u(s)) \mathrm{d} s, \quad t \in[0, b] .
$$

It is easy to see that the fixed point of $Q_{n}$ is the mild solution of nonlocal problem (3.1). Subsequently, we will prove that $Q_{n}$ has a fixed point by using Schauder's fixed point theorem. From assumption (Hf), $(\mathrm{Hg})$, it is easy to check that the mapping $Q_{n}: C([0, b], X) \rightarrow$ $C([0, b], X)$ is continuous and maps $W_{r}$ into itself. According to Schauder's fixed point theorem, it remains to prove that $Q_{n}$ is compact in $W_{r}$.

Firstly, for any $B \subset W_{r}$, we will show that $K_{n} B$ is equicontinuous on $t \in[0, b]$. To this end, let $u \in B$. If $t=0$, then for any $h>0$,

$$
\begin{aligned}
& \left\|\left(Q_{n} u\right)(h)-\left(Q_{n} u\right)(0)\right\| \\
& =\left\|S_{\alpha}(h) S_{\alpha}\left(\frac{1}{n}\right) g(u)+\int_{0}^{h} S_{\alpha}(h-s) f(s, u(s)) \mathrm{d} s-S_{\alpha}\left(\frac{1}{n}\right) g(u)\right\| \\
& \leq\left\|\left(S_{\alpha}(h) S_{\alpha}\left(\frac{1}{n}\right)-S_{\alpha}\left(\frac{1}{n}\right)\right) g(u)\right\|+\int_{0}^{h}\left\|S_{\alpha}(h-s) f(s, u(s))\right\| \mathrm{d} s \\
& \leq\left\|\left(S_{\alpha}(h) S_{\alpha}\left(\frac{1}{n}\right)-S_{\alpha}\left(h+\frac{1}{n}\right)\right) g(u)\right\|+\left\|\left(S_{\alpha}\left(h+\frac{1}{n}\right)-S_{\alpha}\left(\frac{1}{n}\right)\right) g(u)\right\| \\
& \quad+\int_{0}^{h}\left\|S_{\alpha}(h-s) f(s, u(s))\right\| \mathrm{d} s .
\end{aligned}
$$

By Lemma 2.6, we find that $Q_{n} B$ is equicontinuous at $t=0$.

If $t \in(0, b]$, for any $\epsilon>0$, with $t-\epsilon>0$ and $0<|h|<\epsilon$, it follows from (Hf) that

$$
\begin{aligned}
\left\|\left(Q_{n} u\right)(t+h)-\left(Q_{n} u\right)(t)\right\|= & \| S_{\alpha}(t+h) S_{\alpha}\left(\frac{1}{n}\right) g(u)+\int_{0}^{t+h} S_{\alpha}(t+h-s) f(s, u(s)) \mathrm{d} s \\
& -S_{\alpha}(t) S_{\alpha}\left(\frac{1}{n}\right) g(u)-\int_{0}^{t} S_{\alpha}(t-s) f(s, u(s)) \mathrm{d} s \| \\
\leq & \left\|\left(S_{\alpha}(t+h)-S_{\alpha}(t)\right) S_{\alpha}\left(\frac{1}{n}\right) g(u)\right\| \\
& +\int_{0}^{t}\left\|\left(S_{\alpha}(t+h-s)-S_{\alpha}(t-s)\right) f(s, u(s))\right\| \mathrm{d} s \\
& +\int_{t}^{t+h}\left\|S_{\alpha}(t+h-s) f(s, u(s))\right\| \mathrm{d} s \\
\leq & \left\|\left(S_{\alpha}(t+h)-S_{\alpha}(t)\right) S_{\alpha}\left(\frac{1}{n}\right) g(u)\right\| \\
& +\int_{0}^{t-\epsilon}\left\|S_{\alpha}(t+h-s)-S_{\alpha}(t-s)\right\|\|f(s, u(s))\| \mathrm{d} s \\
& +\int_{t-\epsilon}^{t}\left\|S_{\alpha}(t+h-s)-S_{\alpha}(t-s)\right\|\|f(s, u(s))\| \mathrm{d} s \\
& +\int_{t}^{t+h}\left\|S_{\alpha}(t+h-s)\right\|\|f(s, u(s))\| \mathrm{d} s
\end{aligned}
$$




$$
\begin{aligned}
\leq & \left\|\left(S_{\alpha}(t+h)-S_{\alpha}(t)\right) S_{\alpha}\left(\frac{1}{n}\right) g(u)\right\| \\
& +\left\|\rho_{r}\right\|_{L^{1}} \sup _{s \in[0, t-\epsilon]}\left\|\left(S_{\alpha}(t+h-s)-S_{\alpha}(t-s)\right)\right\| \\
& +2 M \int_{t-\epsilon}^{t} \rho_{r}(s) \mathrm{d} s+M \int_{t}^{t+h} \rho_{r}(s) \mathrm{d} s .
\end{aligned}
$$

Note that, by Lemma 2.6(i), we may infer that

$$
\left\|\left(S_{\alpha}(t+h)-S_{\alpha}(t)\right) S_{\alpha}\left(\frac{1}{n}\right) g(u)\right\| \rightarrow 0, \quad \text { as } h \rightarrow 0
$$

uniformly for all $u \in B$. Therefore, from the arbitrariness of $\epsilon$, we can conclude that $Q_{n} B$ is equicontinuous on $[0, b]$.

Secondly, we shall demonstrate that $Q_{n} B(t)$ is relatively compact in $X$ for all $t \in[0, b]$, where $B(t)=\{u(t), u \in B\}, t \in[0, b]$. If $t=0$, then $Q_{n} B(0)=\left\{S_{\alpha}\left(\frac{1}{n}\right) g(u): u \in B\right\}$. Since $S_{\alpha}(t)$, $t>0$ is compact and the condition (Hg) holds, it is obvious that $Q_{n} B(0)$ is relatively compact in $X$. If $t>0$, for any $0<\epsilon<t$, set

$$
\left(Q_{n}^{\epsilon} u\right)(t)=S_{\alpha}(t) S_{\alpha}\left(\frac{1}{n}\right) g(u)+S_{\alpha}(\epsilon) \int_{0}^{t-\epsilon} S_{\alpha}(t-s-\epsilon) f(s, u(s)) \mathrm{d} s
$$

Further, $S_{\alpha}(t)$ is compact at $t>0$, then $Q_{n}^{\epsilon} B(t)$ is relatively compact at $t>0$. In the following, we shall verify that

$$
\left\|Q_{n} u-Q_{n}^{\epsilon} u\right\| \rightarrow 0, \quad \text { as } \epsilon \rightarrow 0,
$$

uniformly for all $u \in B$. In fact, for arbitrary $\epsilon<\delta<t$, we have

$$
\begin{aligned}
\| S_{\alpha}(\epsilon) & \int_{0}^{t-\epsilon} S_{\alpha}(t-s-\epsilon) f(s, u(s)) \mathrm{d} s-\int_{0}^{t-\epsilon} S_{\alpha}(t-s) f(s, u(s)) \mathrm{d} s \| \\
\leq & \int_{0}^{t-\delta}\left\|S_{\alpha}(\epsilon) S_{\alpha}(t-s-\epsilon)-S_{\alpha}(t-s)\right\| \rho_{r}(s) \mathrm{d} s \\
\quad & \quad \int_{t-\delta}^{t-\epsilon}\left\|S_{\alpha}(\epsilon) S_{\alpha}(t-s-\epsilon)-S_{\alpha}(t-s)\right\| \rho_{r}(s) \mathrm{d} s \\
\leq & \int_{0}^{t-\delta}\left\|S_{\alpha}(\epsilon) S_{\alpha}(t-s-\epsilon)-S_{\alpha}(t-s)\right\| \rho_{r}(s) \mathrm{d} s+\left(M^{2}+M\right) \int_{t-\delta}^{t} \rho_{r}(s) \mathrm{d} s .
\end{aligned}
$$

From Lemma 2.6(ii), we know

$$
S_{\alpha}(\epsilon) S_{\alpha}(t-s-\epsilon)-S_{\alpha}(t-s) \rightarrow 0, \quad \text { as } \epsilon \rightarrow 0 \text { for } s \in[0, t-\delta]
$$

Then, from the Lebesgue dominated convergence theorem and the arbitrariness of $\delta$ that

$$
\lim _{\epsilon \rightarrow 0}\left\|S_{\alpha}(\epsilon) \int_{0}^{t-\epsilon} S_{\alpha}(t-s-\epsilon) f(s, u(s)) \mathrm{d} s-\int_{0}^{t-\epsilon} S_{\alpha}(t-s) f(s, u(s)) \mathrm{d} s\right\|=0
$$


Moreover,

$$
\begin{aligned}
& \left\|\left(Q_{n} u\right)(t)-\left(Q_{n}^{\epsilon} u\right)(t)\right\| \\
& =\left\|S_{\alpha}(\epsilon) \int_{0}^{t-\epsilon} S_{\alpha}(t-s-\epsilon) f(s, u(s)) \mathrm{d} s-\int_{0}^{t} S_{\alpha}(t-s) f(s, u(s)) \mathrm{d} s\right\| \\
& \leq\left\|S_{\alpha}(\epsilon) \int_{0}^{t-\epsilon} S_{\alpha}(t-s-\epsilon) f(s, u(s)) \mathrm{d} s-\int_{0}^{t-\epsilon} S_{\alpha}(t-s) f(s, u(s)) \mathrm{d} s\right\| \\
& \quad+\left\|\int_{0}^{t-\epsilon} S_{\alpha}(t-s) f(s, u(s)) \mathrm{d} s-\int_{0}^{t} S_{\alpha}(t-s) f(s, u(s)) \mathrm{d} s\right\| \\
& \leq\left\|S_{\alpha}(\epsilon) \int_{0}^{t-\epsilon} S_{\alpha}(t-s-\epsilon) f(s, u(s)) \mathrm{d} s-\int_{0}^{t-\epsilon} S_{\alpha}(t-s) f(s, u(s)) \mathrm{d} s\right\| \\
& \quad+M \int_{t-\epsilon}^{t} \rho_{r}(s) \mathrm{d} s .
\end{aligned}
$$

Hence, for fixed $n \geq 1$, we can derive by applying (3.2) that

$$
\lim _{\epsilon \rightarrow 0}\left\|\left(Q_{n} u\right)(t)-\left(Q_{n}^{\epsilon} u\right)(t)\right\|=0
$$

Therefore, $Q_{n} B(t)$ is relatively compact in $X$ for all $t \in[0, b]$. By Schauder's fixed point theorem, the operator $Q_{n}$ has a fixed point in $W_{r}$.

Now, define the solution set $D$ and $D(t)$ by

$$
\begin{aligned}
& D=\left\{u_{n} \in C([0, b], X): u_{n}=Q_{n} u_{n}, n \geq 1\right\}, \\
& D(t)=\left\{u_{n}(t): u_{n} \in D, n \geq 1\right\}, \quad t \in[0, b] .
\end{aligned}
$$

Lemma 3.3 Assume that all the conditions in Theorem 3.1 are satisfied. Then for each $t \in[0, b], D(t)$ is relatively compact in $X$ and $D$ is equicontinuous on $(0, b]$.

Proof For $u_{n} \in D, n \geq 1$, we have

$$
u_{n}(t)=S_{\alpha}(t) S_{\alpha}\left(\frac{1}{n}\right) g\left(u_{n}\right)+\int_{0}^{t} S_{\alpha}(t-s) f\left(s, u_{n}(s)\right) \mathrm{d} s, \quad t \in[0, b] .
$$

Let $t \in(0, b], \epsilon>0, u_{n} \in D, n \geq 1$. From hypothesis (Hf), there exists $h \in(0, t)$ such that

$$
\begin{aligned}
\left\|u_{n}(t)-S_{\alpha}(h) u_{n}(t-h)\right\| & \| S_{\alpha}(t) S_{\alpha}\left(\frac{1}{n}\right) g\left(u_{n}\right)+\int_{0}^{t} S_{\alpha}(t-s) f\left(s, u_{n}(s)\right) \mathrm{d} s \\
& \quad-S_{\alpha}(h) S_{\alpha}(t-h) S_{\alpha}\left(\frac{1}{n}\right) g\left(u_{n}\right)-S_{\alpha}(h) \int_{0}^{t-h} S_{\alpha}(t-h-s) f\left(s, u_{n}(s)\right) \mathrm{d} s \| \\
\leq & M\left\|\left(S_{\alpha}(t)-S_{\alpha}(t-h) S_{\alpha}(h)\right) g\left(u_{n}\right)\right\| \\
& +\left\|\int_{0}^{t-h}\left(S_{\alpha}(t-s)-S_{\alpha}(h) S_{\alpha}(t-h-s)\right) f\left(s, u_{n}(s)\right) \mathrm{d} s\right\|
\end{aligned}
$$




$$
\begin{aligned}
& +\left\|\int_{t-h}^{t} S_{\alpha}(t-s) f\left(s, u_{n}(s)\right) \mathrm{d} s\right\| \\
\leq & M\left\|\left(S_{\alpha}(t)-S_{\alpha}(t-h) S_{\alpha}(h)\right) g\left(u_{n}\right)\right\|+\int_{0}^{t-h}\left\|S_{\alpha}(t-s)-S_{\alpha}(h) S_{\alpha}(t-h-s)\right\| \rho_{r}(s) \mathrm{d} s \\
& +M \int_{t-h}^{t} \rho_{r}(s) \mathrm{d} s .
\end{aligned}
$$

Note that, from Lemma 2.6(iii), we have

$$
\begin{aligned}
& \left\|S_{\alpha}(t)-S_{\alpha}(t-h) S_{\alpha}(h)\right\| \rightarrow 0, \quad t>0, \\
& \left\|S_{\alpha}(t-s)-S_{\alpha}(h) S_{\alpha}(t-h-s)\right\| \rightarrow 0, \quad s \in[0, t-h],
\end{aligned}
$$

uniformly as $h \rightarrow 0$. Thus, for any $n \geq 1$, we can conclude that

$$
\lim _{h \rightarrow 0}\left\|u_{n}(t)-S_{\alpha}(h) u_{n}(t-h)\right\|=0 .
$$

Then $D(t), t \in(0, b]$, is precompact since $S_{\alpha}(h), h>0$, is compact.

Finally, we will show that $D$ is equicontinuous for $t \in(0, b]$. Similar to the proof Lemma 3.2, for any $\epsilon>0$, with $t-\epsilon>0$ and $0<|h|<\epsilon$, we have

$$
\begin{aligned}
\left\|u_{n}(t+h)-u_{n}(t)\right\| \leq & \left\|\left(S_{\alpha}(t+h)-S_{\alpha}(t)\right) S_{\alpha}\left(\frac{1}{n}\right) g\left(u_{n}\right)\right\| \\
& +\int_{0}^{t-\epsilon}\left\|S_{\alpha}(t+h-s)-S_{\alpha}(t-s)\right\|\left\|f\left(s, u_{n}(s)\right)\right\| \mathrm{d} s \\
& +\int_{t-\epsilon}^{t}\left\|S_{\alpha}(t+h-s)-S_{\alpha}(t-s)\right\|\left\|f\left(s, u_{n}(s)\right)\right\| \mathrm{d} s \\
& +\int_{t}^{t+h}\left\|S_{\alpha}(t+h-s)\right\|\left\|f\left(s, u_{n}(s)\right)\right\| \mathrm{d} s \\
\leq & \left\|\left(S_{\alpha}(t+h)-S_{\alpha}(t)\right) S_{\alpha}\left(\frac{1}{n}\right) g\left(u_{n}\right)\right\| \\
& +\left\|\rho_{r}\right\|_{L^{1}} \sup _{s \in[0, t-\epsilon]}\left\|S_{\alpha}(t+h-s)-S_{\alpha}(t-s)\right\| \\
& +2 M \int_{t-\epsilon}^{t} \rho_{r}(s) \mathrm{d} s+M \int_{t}^{t+h} \rho_{r}(s) \mathrm{d} s .
\end{aligned}
$$

From Lemma 2.6(i), we know

$$
\begin{aligned}
& S_{\alpha}(t+h)-S_{\alpha}(t) \rightarrow 0, \quad \text { as } h \rightarrow 0, \\
& S_{\alpha}(t+h-s)-S_{\alpha}(t-s) \rightarrow 0, \quad \text { as } h \rightarrow 0 \text { for } s \in[0, t-\epsilon] .
\end{aligned}
$$

Then it follows from the arbitrariness of $\epsilon$ that $\lim _{h \rightarrow 0}\left\|u_{n}(t+h)-u_{n}(t)\right\|=0$, i.e., $D$ is equicontinuous on $(0, b]$.

Proof of Theorem 3.1 To prove that the solution set $D$ of nonlocal problem (3.1) is precompact in $C([0, b], X)$, we should only prove that $D(0)$ is relatively compact in $X$ and $D$ is equicontinuous at $t=0$ due to Lemmas 3.2, 3.3. 
For $u_{n} \in D$, set

$$
\bar{u}_{n}(t)= \begin{cases}u_{n}(t), & t \in[\delta, b] \\ u_{n}(\delta), & t \in[0, \delta]\end{cases}
$$

where $\delta$ comes from the condition (Hg). Then, by condition $(\mathrm{Hg}), g\left(u_{n}\right)=g\left(\bar{u}_{n}\right)$.

At the same time, by Lemma 3.3, without loss of generality, we may suppose that $\bar{u}_{n} \rightarrow$ $u \in C([0, b], X)$, as $n \rightarrow \infty$. Since

$$
\begin{aligned}
\left\|u_{n}(0)-g(u)\right\| & =\left\|S_{\alpha}\left(\frac{1}{n}\right) g\left(u_{n}\right)-g(u)\right\| \\
& \leq\left\|S_{\alpha}\left(\frac{1}{n}\right) g\left(u_{n}\right)-S_{\alpha}\left(\frac{1}{n}\right) g(u)\right\|+\left\|S_{\alpha}\left(\frac{1}{n}\right) g(u)-g(u)\right\| \\
& \leq M\left\|g\left(\bar{u}_{n}\right)-g(u)\right\|+\left\|S_{\alpha}\left(\frac{1}{n}\right) g(u)-g(u)\right\| \\
& \rightarrow 0, \quad n \rightarrow \infty,
\end{aligned}
$$

i.e., $D(0)$ is relatively compact in $X$.

On the other hand, for $t \in(0, b)$,

$$
\begin{aligned}
\left\|u_{n}(t)-u_{n}(0)\right\| & \leq\left\|S_{\alpha}(t) S_{\alpha}\left(\frac{1}{n}\right) g\left(u_{n}\right)-S_{\alpha}\left(\frac{1}{n}\right) g\left(u_{n}\right)\right\|+\int_{0}^{t}\left\|S_{\alpha}(t-s) f\left(s, u_{n}(s)\right)\right\| \mathrm{d} s \\
& =\left\|\left[S_{\alpha}(t)-I\right] S_{\alpha}\left(\frac{1}{n}\right) g\left(u_{n}\right)\right\|+\int_{0}^{t}\left\|S_{\alpha}(t-s) f\left(s, u_{n}(s)\right)\right\| \mathrm{d} s \\
& \rightarrow 0
\end{aligned}
$$

uniformly as $t \rightarrow 0$ since $D(0)=\left\{S_{\alpha}\left(\frac{1}{n}\right) g\left(u_{n}\right): u_{n} \in D\right\}_{n=1}^{+\infty}$ is relatively compact. Thus, we obtain the result that the set $D \subseteq C([0, b], X)$ is equicontinuous at $t=0$. Therefore, $D$ is precompact in $C([0, b], X)$. Without loss of generality, we may suppose that $u_{n} \rightarrow u^{*} \in$ $C([0, b], X)$ as $n \rightarrow \infty$. By the definition of a mild solution for (3.1), we have

$$
u_{n}(t)=S_{\alpha}(t) S_{\alpha}\left(\frac{1}{n}\right) g\left(u_{n}\right)+\int_{0}^{t} S_{\alpha}(t-s) f\left(s, u_{n}(s)\right) \mathrm{d} s
$$

for $t \in[0, b]$. Taking the limit $n \rightarrow \infty$ in both sides, we obtain

$$
u^{*}(t)=S_{\alpha}(t) g\left(u^{*}\right)+\int_{0}^{t} S_{\alpha}(t-s) f\left(s, u^{*}(s)\right) \mathrm{d} s, \quad 0 \leq t \leq b
$$

which implies that $u^{*}$ is a mild solution of nonlocal problem (1.1).

Remark 3.4 The continuity of the resolvent in the uniform operator topology plays a key role in the proof of our main results. Moreover, the technique of approximate solutions is very important in the proof of Theorem 3.1. The application of it not only allows us to get rid of the compactness of the nonlocal item successfully, but it also lets us solve the problem of the compactness of the solution operator at zero. Finally, the method in this paper also could resolve the fractional differential equations via resolvent operators such 
as the differential inclusion, differential equation with delay and differential equations with impulsive conditions etc.

Corollary 3.5 Assume the hypotheses (HA), $(\mathrm{Hg})$, and $(\mathrm{Hf})$ are true for each $r>0$. Moreover,

$$
\begin{aligned}
& \frac{\|g(u)\|}{\|u\|} \rightarrow 0, \quad \text { as }\|u\| \rightarrow \infty, \\
& \frac{\|f(t, x)\|}{\|x\|} \rightarrow 0, \quad \text { as }\|x\| \rightarrow \infty, \text { uniformly for } t \in[0, b],
\end{aligned}
$$

then the nonlocal problem (1.1) has at least one mild solution in $C([0, b], X)$.

Remark 3.6 The condition (3.3) is satisfied if there exist constant $L_{1}, L_{2}>0$ and $\lambda_{1}, \lambda_{2} \in$ $[0,1)$ such that

$$
\begin{aligned}
& \|g(u)\| \leq L_{1}(1+\|u\|)^{\lambda_{1}}, \\
& \|f(t, x)\| \leq L_{2}(1+\|x\|)^{\lambda_{2}}, \quad t \in[0, b] .
\end{aligned}
$$

Corollary 3.7 Let conditions (HA), (Hf) be satisfied. Suppose that $g(u)=\sum_{j=1}^{p} c_{j} \sqrt[3]{u\left(s_{j}\right)}$, where $c_{j}, j=1,2, \ldots, p$, are given constants, and $0<s_{1}<s_{2}<\cdots<s_{p} \leq b$. Then the nonlocal problem (1.1) has at least one mild solution in $C([0, b], X)$ provided that $M^{2} \sum_{j=1}^{p}\left|c_{j}\right| \sqrt[3]{r}+$ $M\left\|\rho_{r}\right\|_{L^{1}} \leq r$.

Proof It is easy to see that if $g(u)=\sum_{j=1}^{p} c_{j} \sqrt[3]{u\left(s_{j}\right)}$, condition $(\mathrm{Hg})$ holds with $\delta=s_{1}$. Thus all the conditions in Theorem 3.1 are satisfied. Then the nonlocal problem (1.1) has at least one mild solution on $[0, b]$.

\section{Example}

Finally, we give a simple example to illustrate our theory.

Example 4.1 Consider the following fractional partial differential equation:

$$
\begin{aligned}
& D_{t}^{\alpha} w(t, x)=\frac{\partial^{2} w(t, x)}{\partial x^{2}}+\frac{1}{\Gamma(\alpha)} \int_{0}^{t} \frac{\sqrt{w(t, x)}}{(t-s)^{1-\alpha}} \mathrm{d} s, \quad 0<t<1,0<x<\pi, \\
& w(t, 0)=w(t, \pi)=0, \quad 0 \leq t \leq 1, \\
& w(0, x)=u_{0}(x)-\sum_{j=1}^{p} c_{j} \sqrt[3]{w\left(s_{j}, x\right)}, \quad 0<x<\pi, 0<s_{1}<s_{2}<\cdots<s_{p} \leq 1,
\end{aligned}
$$

where $0<\alpha<1, u_{0} \in L^{2}[0, \pi], D_{t}^{\alpha}$ is the Caputo fractional derivative, $c_{j}, j=1,2, \ldots, p$, and $s_{j}, j=1,2, \ldots, p$, are given real numbers.

Take $X=L^{2}[0, \pi]$, with the norm $\|\cdot\|_{2}$, and consider the operator $A: D(A) \subseteq X \rightarrow X$ to be $A x=x^{\prime \prime}$, where $D(A)=\left\{x \in X: x, x^{\prime}\right.$ are absolutely continuous, $\left.x^{\prime \prime} \in X, x(0)=x(\pi)=0\right\}$. It is well known that $A$ is the infinitesimal generator of an analytic semigroup $T(t)$ for $t>0$ on $X$. Furthermore, $A$ has discrete spectrum with eigenvalues of the form $-n^{2}, n \in N$, and corresponding normalized eigenfunctions given by $z_{n}(\xi):=\left(\frac{2}{\pi}\right)^{\frac{1}{2}} \sin (n \xi)$. In addition, 
$\left\{z_{n}: n \in N\right\}$ is an orthonormal basis for $X, T(t) x=\sum_{n=1}^{\infty} e^{-n^{2} t}\left\langle x, z_{n}\right\rangle z_{n}$ for all $x \in X$ and every $t>0$. From this expression it follows that $T(t), t>0$, is a uniformly bounded compact semigroup. Moreover,

$$
\|T(t) x\|_{2}^{2} \leq \sum_{n=1}^{\infty} e^{-2 n^{2} t}\left|\left\langle x, z_{n}\right\rangle\right|^{2} \leq \sum_{n=1}^{\infty}\left|\left\langle x, z_{n}\right\rangle\right|^{2}=\|x\|^{2}
$$

i.e. $\|T(t)\| \leq 1, t>0$.

By the subordination principle [19, Theorem 3.11], we know that $A$ also generates a compact $\alpha$-order fractional analytic resolvent $S_{\alpha}(t)$ of analyticity type $\left(\omega_{0}, \theta_{0}\right)$ for some $\omega_{0}, \theta_{0}$ and

$$
S_{\alpha}(t)=\int_{0}^{\infty} \Phi_{\alpha}(s) T\left(s t^{\alpha}\right) \mathrm{d} s, \quad t>0,
$$

where

$$
\Phi_{\gamma}(s):=\sum_{n=0}^{\infty} \frac{(-s)^{n}}{n ! \Gamma(-\gamma n+1-\gamma)}, \quad 0<\gamma<1,
$$

is the Wright function. First, we prove that there exists a constant $M>0$ such that $\left\|S_{\alpha}(t)\right\| \leq M, t \in[0,1]$. In fact, by (4.2), we have

$$
\left\|S_{\alpha}(t)\right\| \leq \int_{0}^{\infty}\left\|\Phi_{\alpha}(s) T\left(s t^{\alpha}\right)\right\| \mathrm{d} s \leq \int_{0}^{\infty} \Phi_{\alpha}(s) \mathrm{d} s=1
$$

Therefore, $\left\|S_{\alpha}(t)\right\| \leq 1, t>0$.

We now assume that:

(1) $f:[0,1] \times X \rightarrow X$ is a continuous function defined by

$$
f(t, z)(x)=\sqrt{z}, \quad 0 \leq t \leq 1,0 \leq x \leq \pi,
$$

(2) $g: C([0,1], X) \rightarrow X$ is a continuous function defined by

$$
g(u)(x)=u_{0}(x)-\sum_{j=1}^{p} c_{j} \sqrt[3]{u\left(s_{j}\right)(x)}, \quad 0 \leq x \leq \pi, 0<s_{1}<s_{2}<\cdots<s_{p} \leq 1
$$

where $u(s)(x)=w(s, x), 0 \leq s \leq 1,0 \leq x \leq \pi$. Moreover, for each $l>0$, we have $\|f(t, z)\| \leq \sqrt{l}$ for $t \in[0,1]$, and all $\|z\| \leq l$.

Under the above conditions, the problem (4.1) also can be reformulated as the abstract problem (1.1), and conditions (Hg), (Hf) are satisfied with $\rho_{l}=\sqrt{l}, \delta=s_{1}$. On the other hand, there must be $r>0$ such that the inequality $\left\|u_{0}\right\|_{2}+\sum_{j=1}^{p} c_{j} \sqrt[3]{r}+\sqrt{r} \leq r$ holds, then, according to Theorem 3.1, the problem (4.1) has at least one mild solution on $[0,1]$. 


\section{Author details}

${ }^{1}$ Department of Applied Mathematics, Shanxi University of Finance and Economics, Taiyuan, Shanxi 030006, P.R. China.

${ }^{2}$ Department of Mathematics, Changshu Institute of Technology, Suzhou, Jiangsu 215500, P.R. China. ${ }^{3}$ Department of Mathematics, Yangzhou University, Yangzhou, Jiangsu 225002, P.R. China.

\section{Acknowledgements}

The work was supported by the National Science Foundation of China $(11001034,11271316)$ and the Qing Lan Project of Jiangsu Province of China.

Received: 27 June 2014 Accepted: 8 September 2014 Published: 24 Sep 2014

\section{References}

1. Byszewski, L, Lakshmikantham, V: Theorem about the existence and uniqueness of solutions of a nonlocal Cauchy problem in a Banach space. Appl. Anal. 40, 11-19 (1990)

2. Byszewski, L: Theorems about the existence and uniqueness of solutions of a semilinear evolution nonlocal Cauchy problems. J. Math. Anal. Appl. 162(2), 494-505 (1991)

3. Ntouyas, SK, Tsamatos, PC: Global existence for semilinear evolution equations with nonlocal conditions. J. Math. Anal. Appl. 210(2), 679-687 (2009)

4. Fan, Z, Li, G: Existence results for semilinear differential equations with nonlocal and impulsive conditions. J. Funct. Anal. 258, 1709-1727 (2010)

5. Aizicovici, S, Staicu, V: Multivalued evolution equations with nonlocal initial conditions in Banach spaces. Nonlinear Differ. Equ. Appl. 14, 361-376 (2007)

6. Liang, J, Liu, J, Xiao, T: Nonlocal impulsive problems for nonlinear differential equations in Banach spaces. Math. Comput. Model. 49, 798-804 (2009)

7. Mophou, GM, N'Guerekata, GM: Existence of the mild solution for some fractional differential equations with nonlocal conditions. Semigroup Forum 79(2), 315-322 (2009)

8. Zhu, L, Li, G: On a nonlocal problem for semilinear differential equations with upper semicontinuous nonlinearities in general Banach spaces. J. Math. Anal. Appl. 341, 660-675 (2008)

9. Zhu, L, Huang, Q, Li, G: Existence results of semilinear differential equations with nonlocal initial conditions in Banach spaces. Nonlinear Anal. 74, 5133-5140 (2011)

10. Agarwal, RP, Andrade, B, Cuevas, C: On type of periodicity and ergodicity to a class of fractional order differential equations. Adv. Differ. Equ. 2010, Article ID 179750 (2010)

11. Agarwal, RP, Santos, JP, Cuevas, C: Analytic resolvent operator and existence results for fractional integrodifferential equations. J. Abstr. Differ. Equ. Appl. 2(2), 26-47 (2012)

12. Agarwal, RP, Cuevas, C, Soto, H, El-Gebeily, M: Asymptotic periodicity for some evolution equations in Banach spaces. Nonlinear Anal. 74, 1769-1798 (2011)

13. Diethelm, K, Freed, AD: On the solution of nonlinear fractional-order equations used in the modeling of viscoplasticity. In: Scientific Computing in Chemical Engineering II, pp. 217-224. Springer, Heidelberg (1999)

14. Cuevas, C, Sepulveda, A, Soto, H: Almost periodic pseudo-almost periodic solutions to fractional differential and integro-differential equations. Appl. Math. Comput. 218, 1735-1745 (2011)

15. El-Sayed, AMA: Fractional-order diffusion-wave equation. Int. J. Theor. Phys. 35(2), 311-322 (1996)

16. Cuevas, C, Pierri, M, Sepulveda, A: Weighted S-asymptotically $\omega$-periodic solutions of a class of fractional differential equations. Adv. Differ. Equ. 2011, Article ID 584874 (2011)

17. Cuevas, C, Lizama, C, Soto, H: Asymptotic periodicity for strongly damped wave equations. Abstr. Appl. Anal. 2013, Article ID 308616 (2013)

18. Sebaa, N, Fellah, ZEA, Lauriks, W, Depollier, C: Application of fractional calculus to ultrasonic wave propagation in human cancellous bone. Signal Process. 86, 2668-2677 (2006)

19. Fan, Z: Characterization of compactness for resolvents and its applications. Appl. Math. Comput. 232, 60-67 (2014)

20. Miller, KS, Ross, B: An Introduction to the Fractional Calculus and Fractional Differential Equations. pp. 1-37. Wiley, New York (1993)

21. Li, K, Peng, J, Jia, J: Cauchy problems for fractional differential equations with Riemann-Liouville fractional derivatives. J. Funct. Anal. 263, 476-510 (2012)

22. Chen, L, Fan, Z: On mild solutions to fractional differential equations with nonlocal conditions. Electron. J. Qual. Theory Differ. Equ. 2011, 53 (2011)

23. Xue, X: Existence of semilinear differential equations with nonlocal initial conditions. Acta Math. Sin. 23(6), 983-988 (2007)

24. Podlubny, I: Fractional Differential Equations. Academic Press, San Diego (1999)

25. Prüss, J: Evolutionary Integral Equations and Applications. Birkhäuser, Basel (1993)

10.1186/1687-1847-2014-251

Cite this article as: Chen et al.: On a nonlocal problem for fractional differential equations via resolvent operators. Advances in Difference Equations 2014, 2014:251 